\title{
Cylindrical Echocardiographic Image Segmentation Based on 3D Deformable Models
}

\author{
J. Montagnat, H. Delingette, and G. Malandain \\ INRIA Sophia, Epidaure project, BP 93 \\ 2004 route des Lucioles, 06902 Sophia-Antipolis Cedex France \\ http://www-sop.inria.fr/epidaure/
}

\begin{abstract}
This paper presents a 3D echocardiographic image segmentation procedure based on deformable surfaces. We first propose to adapt filtering techniques to the cylindrical geometry of several 3D ultrasound image devices. Then we compare the effect of different external forces on a surface template deformation inside volumetric echocardiographic images. An original method involving region grey-level analysis along the model normal directions is described. We rely on an a priori knowledge of the cardiac left ventricle shape and on region grey-level values to perform a robust segmentation. During the deformation process the allowable surface deformation is modified. Finally, we show experimental results on very challenging sparse and noisy images and quantitative measurements of the left ventricle volume.
\end{abstract}

\section{Introduction}

Since its introduction over the past few years, 3D ultrasound imagery has been very promising due to its low cost and non-invasive nature. Furthermore, the development of real-time 3D ultrasound probes reveals numerous applications for the diagnosis and therapy of patients. Cardiac left ventricle modeling permits a quantitative computation of the ventricle ejection fraction which is of major importance in detecting heart pathologies. In this paper, we propose a 3D reconstruction algorithm of the left ventricle based on 3D deformable models.

In many cases, 3D ultrasound systems are based on a mobile 2D probe acquiring along non-aligned planes [13]. In this paper, we consider rotative probes producing a set of $2 \mathrm{D}$ planes intersecting each other along a revolution axis. These images are acquired with a cylindrical geometry implying that the density of information decreases as a function of the distance to the axis (figure 1).

Previous work of Winterfeldt [14] and Jacob [6] have mainly considered 2D image segmentation and tracking approaches without any spatial coherence between slices. Statistical information (mainly principal component analysis) has also been used to restrain the allowable deformations of a model $[6,4]$. Several authors proposed to reduce speckle level by spatial compounding [11] or to detect boundaries in US images by modeling speckle noise [1].

In this paper, we propose to use "naive" image processing techniques based on rough approximations of the image speckle and noise. The introduction of 


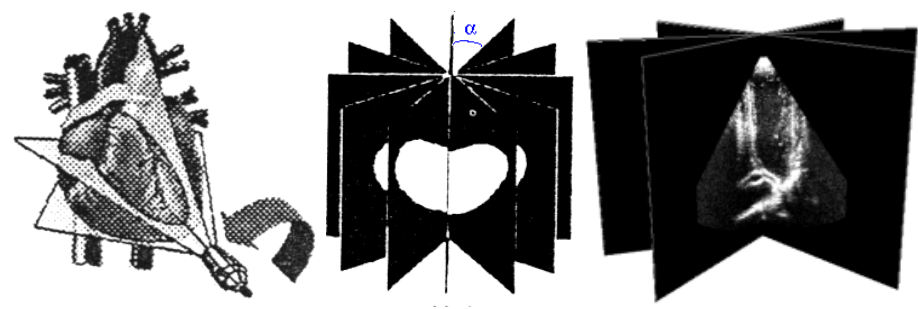

Fig. 1. The rotative probe and the resulting US images with cylindrical symmetry.

3D deformation and some basic a priori knowledge about the data's reliability compensate for the presence of outliers and increase the robustness of the image segmentation. The main contributions of our approach are to consider the full 3D problem with 3D model deformations inside a volumetric image, to adapt image filtering techniques to the ultrasound images cylindrical geometry, and to use a region-based approach for finding the boundaries of the left ventricle.

\section{Simplex Meshes}

A 2-simplex mesh is a discrete non-parametric representation of a surface in $\mathbb{R}^{3}$ defined by a set of vertices $\left\{\mathbf{V}_{i}\right\}_{i}$ and their connectivity. It is a regular 3connected mesh, its topology is dual to a triangulation, and it can represent surfaces of any topology. The geometry of simplex meshes allows one to define discrete quantities such as mean curvature at each vertex (see [2] for details). All vertices follow a Newtonian law of motion. Three kinds of forces are computed at each vertex $\mathbf{V}_{i}$. The internal force $f_{i}^{\text {int }}$ enforces some regularization behavior (such as $\mathrm{C}^{2}$ continuity). The external force $f_{i}^{e x t}$ pulls the model towards the ventricle boundaries. The global displacement force $f_{i}^{g l o b a l}$ is computed from a global transformation with few degrees of freedom designed to constrain all the vertices displacements. The law of motion is discretized using finite differences and an explicit iterative scheme:

$$
\mathbf{V}_{i}^{t+1}=\mathbf{V}_{i}^{t}+(1-\gamma)\left(\mathbf{V}_{i}^{t}-\mathbf{V}_{i}^{t-1}\right)+\alpha f_{i}^{i n t}+\beta\left(\lambda f_{i}^{\text {ext }}+(1-\lambda) f_{i}^{\text {global }}\right),
$$

where $\mathbf{V}_{i}^{t}$ denotes the position of vertex $\mathbf{V}_{i}$ at time $t, \gamma$ is a damping coefficient, $\alpha$ and $\beta$ are the internal and external force weights, and $\lambda$ is a locality weight. The time step is hidden in the $\alpha, \beta$ and $\gamma$ coefficients. We use a refinement approach, limiting model deformation capabilities by setting $\lambda$ to zero at the beginning of the deformation process, then letting it gradually increase to allow more deformations. The $\alpha$ coefficient value is set to 1 thus having $\beta$ variations weight the respective influence of the internal and the external forces. The $\beta$ coefficient varies from 1 to 0.1 as $\lambda$ increases. The damping value $\gamma$ is fixed to 0.35 based on some empirical results. An automatic algorithm for governing $\lambda$ evolution was proposed in [10]. 


\section{Gradient Computation in Cylindrical Geometry}

In 3D images based on a regular lattice (Cartesian images), the computation of gradient vectors is mostly based on separable filters (e.g., Sobel operators) or recursive filtering (e.g., Canny-Deriche filters [9]). When considering images of cylindrical geometry, classical approaches can be used in each slice, computing $2 \mathrm{D}$ gradients and neglecting the tangential component. It is also possible to interpolate the image on a regular lattice before applying 3D operators. Unfortunately, this approach is hopeless due to the important angular resolution and the low signal-to-noise ratio. Instead, we compute a $3 \mathrm{D}$ gradient vector for each image voxel in the cylindrical geometry (see [5] for a similar approach in 2D). Let $M_{0}$ be a point in Euclidean space with Cartesian coordinates $\left(x_{0}, y_{0}, z_{0}\right)$ and cylindrical coordinates $\left(r_{0}, \theta_{0}, z_{0}\right)$ (see figure 2 left). Let $I$ be the cylindrical image defined as $I: B \subset \mathbb{R}^{3} \rightarrow \mathbb{R}$, where $B$ is the discrete cylindrical grid over which the grey-level values are known. The convolution of a $3 \mathrm{D}$ signal $S$ by a filter $f$ at point $M_{0}$ is defined in Cartesian space as:

$$
(S \otimes f)\left(M_{0}\right)=\int_{-\infty}^{\infty} \int_{-\infty}^{\infty} \int_{-\infty}^{\infty} S\left(x_{0}-x, y_{0}-y, z_{0}-z\right) f(x, y, z) d x d y d z .
$$

To express this equation in cylindrical space we use the coordinate transformation $x=r \cos (\theta), y=r \sin (\theta)$, and $z=z$. Let $J$ be the Jacobian matrix corresponding to this transformation. A variable change in equation 1 leads to:

$(S \otimes f)\left(M_{0}\right)=\int_{-\infty}^{\infty} \int_{0}^{2 \pi} \int_{0}^{\infty} S\left(x_{0}-r \cos (\theta), y_{0}-r \sin (\theta), z_{0}-z\right) f(x, y, z)|J| d r d \theta d z$.

Since image $I$ is a discrete signal, we discretize equation 2. Let $\delta r, \delta \theta$, and $\delta z$ be the dimensions of the discrete filter to apply to $I$, the filtered value of $I$ at point $M_{0} \in B$, knowing that $|J|=r$, is:

$(I \otimes f)\left(M_{0}\right)=\sum_{z=z_{0}-\delta z}^{z_{0}+\delta z} \sum_{\theta=\theta_{0}-\delta \theta}^{\theta_{0}+\delta \theta} \sum_{r=r_{0}-\delta r}^{r_{0}+\delta r}-I(r, \theta, z) r f\left(x_{0}-r \cos (\theta), y_{0}-r \sin (\theta), z_{0}-z\right)$.

We use Deriche filters [3] to perform gradient computation in cylindrical geometry. Let $D_{d}$ and $S_{d}$ be the mono-dimensional Deriche derivative and smoothing filters respectively, in dimension $d \in\{x, y, z\}: D_{d}(u)=u e^{-\alpha|u|}$ and $S_{d}(u)=$ $(\alpha|u|+1) e^{-\alpha|u|}$. The directional gradient operators are defined as $G_{x}(u)=$ $D_{x}(u) S_{y}(u) S_{z}(u), G_{y}(u)=S_{x}(u) D_{y}(u) S_{z}(u)$, and $G_{z}(u)=S_{x}(u) S_{y}(u) D_{z}(u)$. The discrete masks are computed by sampling $G_{x}, G_{y}$, and $G_{z}$ values then normalizing mask coefficients such that the sum of positive coefficients equals 1 and the sum of negative coefficients equals -1 . This ensures that the derivative mask coefficients sum is nul.

Figure 2 (center) illustrates the filter response on a slice of a synthetic cube image for a $3^{3}$ (top) and a $7^{3}$ (bottom) filter. Figure 2 (right) compares the output of the 2D Sobel filters (left) and a 3D cylindrical operator (right) in one image plane. The cylindrical operator improves significantly the edge detection and reduces the speckle effect by filtering along the axial direction, especially close to the rotation axis where dense data is available. 


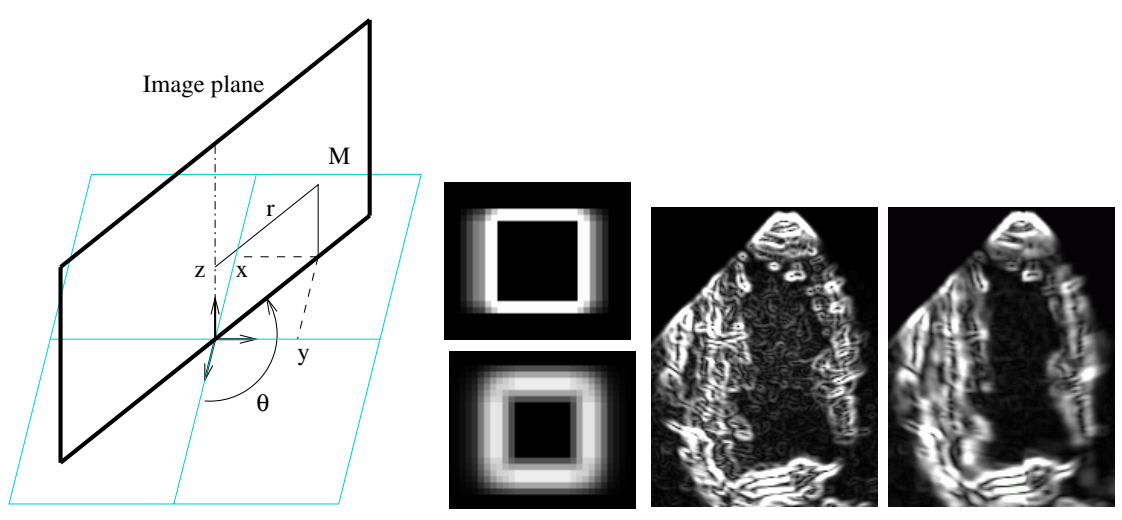

Fig. 2. (left) Cylindrical geometry; (center) cylindrical gradient filter response on a cube; (right) comparison of Sobel operators and the cylindrical gradient filter.

\section{External Forces for 3D US Image Segmentation}

Our deformable simplex mesh relies on external forces for segmenting the left ventricle in 3D US images. These forces should attract each model vertex towards the closest left ventricle boundary voxel. In echocardiographic images several difficulties arise for finding object boundaries. The speckle, inherent to the image formation of US imagery, and the image noise mainly originating from the digitalization process, are responsible for causing false contour detections (false positives). The variable information density, due to the cylindrical geometry of the image, and the missing information, due to the low energy of the ultrasound beam reflected at the organ interfaces and the rib occlusions, make the ventricle contours hard to detect (false negatives). We rely on the model regularizing behavior and the robust force expressions proposed below to deal with the false positives and the false negatives.

\subsection{Scan-Line Algorithm}

The force expressions are computed for each vertex by scanning along the model normal direction until a boundary voxel is reached. Indeed, it has been shown in [8] that the class of surface deformations is not restricted by only considering displacement along each vertex normal. Thus, we restrict the search for boundary points along the normal direction $\mathbf{n}_{i}$ of each vertex $\mathbf{V}_{i}$.

We proceed by scanning all the cylindrical voxels which intersect with the normal line $\left(\mathbf{V}_{i}, \mathbf{n}_{i}\right)$ in the volumetric image within a fixed range centered on each vertex (see figure 3 left). The image intensity $I(\mathbf{P})$ at any normal line point $\mathbf{P}$ is tri-linearly interpolated from the eight closest image voxel intensities $I_{0}$ to $I_{7}$ (see figure 3 center). This algorithm outputs a list of voxels for which we store 
their interpolated intensity value and their Euclidian distance from $\mathbf{V}_{i}$. The scan-line range is fixed as a percentage of the image size. We used a ten-voxel length scan-line in our experiments.
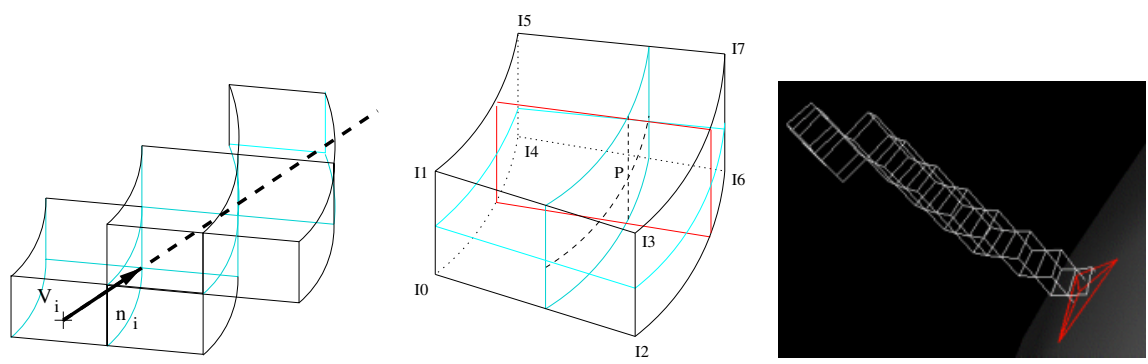

Fig. 3. Normal line and the scanned voxels; Tri-linear interpolation on a cylindrical voxel; 3D display of the scanned voxels.

\subsection{Gradient Norm Extremum}

We first use the extracted scan line in order to find the extremum of the gradient norm along the normal direction. In practice, the normal direction at a gradient vertex quickly converges towards the direction of the gradient vector in presence of a strong boundary. In order to improve this external force computation, we propose to add two additional constraints for a voxel to be considered as the closest boundary voxel. Since the cavity of left ventricle is surrounded by structures that appear brighter, we use a gradient orientation constraint to retain only boundaries whose gradient vector direction is roughly the same that normal direction. We constrain the boundary voxel to belong to a given interval of intensity in order to eliminate spurious boundary information.

\subsection{Region-Based Algorithm}

The previous algorithm may still fail due to the presence of strong edges in the ventricle boundaries vicinity, if such a neighboring edge has a higher gradient than the ventricle edge, and if it meets the two additional constraints listed above. In order to improve the discrimination of the boundary search algorithm, we propose to use both the notion of region and boundary. Region-based image segmentation has been proposed in [12] and has proven to be more powerful than gradient-based approaches. We first extract the intensity profile of a vertex and filter the intensity values to decrease the speckle effect (both Gaussian and Median smoothing have been implemented). The smoothed profile is scanned from the inside to the outside to find a set of consecutive voxels within a given intensity range and of length greater than a minimum threshold. Once the beginning of a region has been found, we search for the first voxel value that does not belong to the region intensity range thus corresponding to the end of the region. 
Given that such a voxel has been found we then look for a voxel whose gradient norm is above a given threshold in the vicinity of the region end. This algorithm requires to set the region intensity range and the gradient norm threshold parameters depending on the segmented images. The minimum region length is a trade-off between computation time and the algorithm accuracy. We used a four-voxel length in our experiments.

\section{Experimental Results}

We first study the effects of the image angular resolution on the model deformation process. A set of synthetic cylindrical slice images was generated by intersecting the geometric face model shown in figure 4 (left) with planes sampled every 20, 10, 5, and 3 degrees. We then deform a spherical model in the $3 \mathrm{D}$ cylindrical images produced. The results are shown in the rest of figure 4 . Unsurprisingly, the retrieved surface shape improves as the angular resolution increases. For low resolution images the model has to be more constrained so that it correctly interpolates sparse data. Forces computed from higher resolution images are more reliable and the a priori model information is less critical in that case.
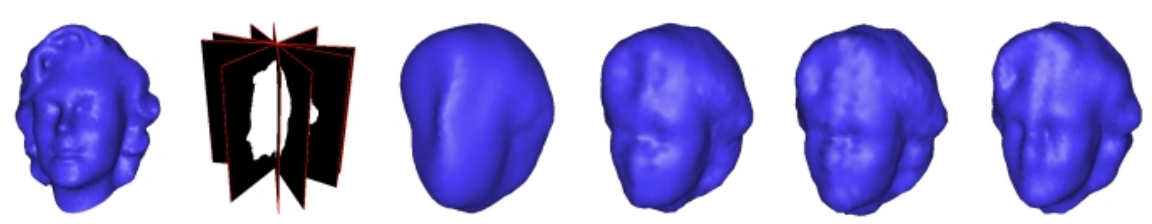

Fig. 4. Face model; Sampled planes; Deformed models for an angular resolution of $20,10,5$, and 3 degrees of an arc.

The next experiment compares the cardiac left ventricle reconstruction from a 3D echocardiographic image using a gradient of the gradient norm guiding force as introduced by Kass et al [7] and the forces introduced in section 4. The model is initialized as an ellipsoide roughly centered inside the ventricle. Figure 5 shows the reconstructed surfaces and the intersection of each surface with one of the image planes. The template image quality is high ( 4 degrees of arc resolution) and gradients are compute using a $7^{3}$ cylindrical filter. The gradient of the gradient norm is not reliable enough due to the large amount of speckle which introduces too many false positives. The scan-line algorithm provides better results for both the gradient extremum and the inner dark regions search. The region approach better smoothes the boundary and is more accurate as can be clearly seen in the mitral valve area.

Finally, we show the temporal evolution of the cardiac left ventricle extracted from a series of eight 3D echocardiographic images. Due to the images'poor 

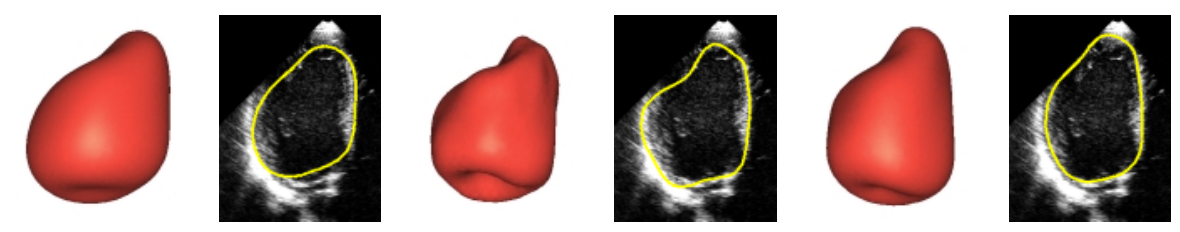

Fig. 5. gradient of the gradient norm (left), gradient extremum (center), and region based (right) reconstruction.

quality (20 degrees of arc resolution and a very high noise level due to video signal digitization) we prefer the more robust region based approach. The model built in the previous experiment is used as a template for the first image segmentation and at each time step, the model is initialized from its position at the previous time instant. Figure 6 shows one out of two reconstructed models from end diastole to end systole (left to right). The intersection of each surface model with three image planes at 20 (second row), 80 (third row), and 140 (fourth row) degrees of arc is shown. The surface model allows for an accurate computation of the ventricle volume variation through the cardiac cycle (figure 6 top, left), and therefore, of the ejection fraction (here we found $38 \%$ ).

\section{Conclusion}

In this paper we demonstrate the ability of $3 \mathrm{D}$ deformable models to segment cylindrical echocardiographic images. The model provides enough intrinsic (shape) and extrinsic (grey-level range) a priori knowledge to constrain the deformations properly even in the presence of very sparse and noisy data. Due to its simplicity, the proposed method is efficient (about 5 minutes to generate the 8 surface models shown in figure 6 on a Digital PWS500). The 3D approach allows an accurate computation of the left ventricle ejection fraction, yet, this model does not take advantage of the time continuity that could be used since the computations are performed off-line. We also plan to improve the region matching algorithm by introducing more accurate knowledge of image grey-level distributions.

\section{Acknowledgments}

We are grateful to Gary Schwartz from ATL Ultrasound, a Philips Medical Systems Company, Marie-Odile Berger from ISA research, Loria, and Dr Lethor, CHU Brabois, for providing the 3D ultrasound images used in this paper. 

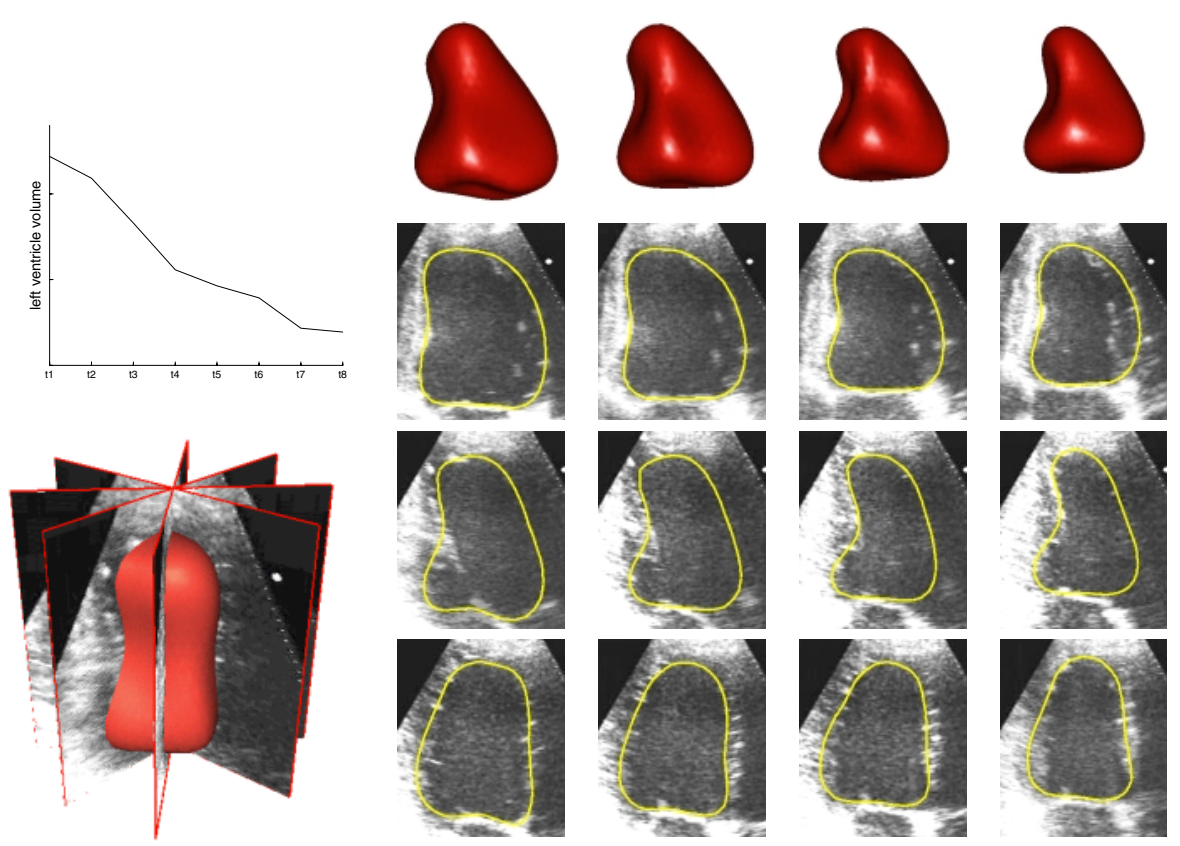

Fig. 6. Four out of the eight instants reconstructed from a $4 \mathrm{D}$ echocardiographic image.

\section{References}

1. R. N. Czerwinski. Line and boundary detection in speckle images. IEEE Transactions on Image Processing, 7(12):1700-1714, 1998. 168

2. H. Delingette. General object reconstruction based on simplex meshes. to appear in International Journal of Computer Vision, 1999. 169

3. R. Deriche. Using Canny's criteria to derive a recursively implemented optimal edge detector. International Journal of Computer Vision, 1(2), 1987. 170

4. A. Giachetti. On-line analysis of echocardiographic image sequences. Medical Image Analysis, 2(3):261-284, 1999. 168

5. I. Herlin and N. Ayache. Feature extraction and analysis methods for sequences of ultrasound images. In Proceedings of the European Conference in Computer Vision (ECCV'92), pages 43-57, 1992. 170

6. G. Jacob, A. Noble, M. Mulet-Parada, and A. Blake. Evaluating a robust contour tracker on echocardiographic sequences. Medical Image Analysis, 3(1):63-75, 1999. 168

7. M. Kass, A. Witkin, and D. Terzopoulos. Snakes: Active Contour Models. International Journal of Computer Vision, 1:321-331, 1988. 173

8. B. Kimia, A. Tannenbaum, and S. Zucker. On the evolution of curves via a function of curvature i. the classical case. Journal of Mathematical Analysis and Applications, 163:438-458, 1992. 171 
9. O. Monga, R. Deriche, G. Malandain, and J.-P. Cocquerez. Recursive filtering and edge tracking: two primary tools for 3-D edge detection. Image and Vision Computing, 9(4):203-214, August 1991. 170

10. J. Montagnat and H. Delingette. Globally constrained deformable models for 3D object reconstruction. Signal Processing, 71(2):173-186, December 1998. 169

11. R. Rohling, A. Gee, and L. Berman. Three-dimensional spatial compounding of ultrasound images. Medical Image Analysis, 1(3):177-193, 1997. 168

12. R. Ronfard. Region-based strategies for active contour models. International Journal of Computer Vision, 13(2):229-251, 1994. 172

13. G. Treece, R. Prager, A. Gee, and L. Berman. Fast surface and volume estimation from non-parallel cross-sections, for freehand 3-d ultrasound. Technical Report 326, Cambridge University Engineering Department, july 1998. 168

14. G. Winterfeldt, MO. Berger, JP. Lethor, and M. Handschuhmacher. Expert Model Based 3D Reconstruction of the left Ventricle Using Transthorasic Echographic Images. In Computers in Cardiology, sept 1997. 168 